CARNETS OE Carnets de géographes

GÉOGRAPHES.

9 | 2016

Géographies des émotions

\title{
Le touriste, l'émotion et la mémoire douloureuse
}

Dominique Chevalier et Isabelle Lefort

\section{(2) OpenEdition}

\section{Journals}

Édition électronique

URL : http://journals.openedition.org/cdg/644

DOI : $10.4000 /$ cdg. 644

ISSN : 2107-7266

Éditeur

UMR 245 - CESSMA

\section{Référence électronique}

Dominique Chevalier et Isabelle Lefort, «Le touriste, l'émotion et la mémoire douloureuse », Carnets de géographes [En ligne], 9 | 2016, mis en ligne le 30 novembre 2016, consulté le 20 avril 2019. URL: http://journals.openedition.org/cdg/644 ; DOI : 10.4000/cdg.644

\section{c) (i) $\Theta$}

La revue Carnets de géographes est mise à disposition selon les termes de la Licence Creative Commons Attribution - Pas d'Utilisation Commerciale - Pas de Modification 4.0 International. 


\title{
LE TOURISTE, L'EMOTION ET LA MEMOIRE DOULOUREUSE
}

\author{
DOMINIQUE CHEVALIER \\ Université Lyon1-ESPÉ, EVS (UMR 5600) \\ Maîtresse de conférence HDR, Géographie \\ Dominique.chevalier@univ-lyon1.fr
}

ISABELLE LEFORT

Université Lyon2, EVS (UMR 5600)

Professeur des universités, Géographie

Isabelle.lefort@univ-lyon2.fr

\begin{abstract}
Résumé
Les lieux de mémoires douloureuses comptent aujourd'hui parmi les destinations prisées d'un tourisme mondialisé. L'article s'attache aux modalités expérientielles et émotionnelles qui participent de l'agencement de leurs visites, en analysant les scénographies et les parcours, mais aussi l'expression des émotions par les touristes comme celle de leur convenance et de leur adéquation aux lieux. Les émotions constituent ainsi un opérateur spatialisé de mémoire, fondé sur l'épreuve et l'expérience des lieux de mémoire douloureuse, mais révélant dans le même espace-temps la tension entre les horizons de collectifs à construire et à commémorer.
\end{abstract}

Mots-clefs : mémoire douloureuse, Shoah, tourisme, émotion, expérience.

\begin{abstract}
:
Nowadays, places linked to painful memories are among the most favourite visiting spots in a world of globalized tourism. This paper deals with the experimental and emotional setups which organize the visits of such places. It endeavours to scrutinize the various scenographies and paths laid out for the tourists' use. II also deals with the expression of emotions by the latter as well as their appropriateness to the places visited. Thus, the emotions can be construed as a spatialized agent of memory, based upon the confrontation with and the discovery of the places of painful memories. As such, they do reveal in the same space-time framework the tension between the expectations of collective experiences to be built and to be commemorated.
\end{abstract}

Keywords: Painful Memories, Shoah/Holocaust, Tourism, Emotion, Experience/Experiment. 
"Sans émotions, il est impossible de transformer les ténèbres en lumière et l'apathie en mouvement » (C.G Jung).

"La constitution des groupes humains a pour racine naturelle la mise en commun d'expériences illusoires » (D. W. Winnicott).

\section{Introduction}

Les tourismes de mémoire (Hertzog, 2012) ${ }^{1}$ sont aujourd'hui parties prenantes d'une offre touristique mondialisée ${ }^{2}$, en miroir d'une " nouvelle sensibilité à l'égard des victimes (qui) éclaire le $20^{\mathrm{e}}$ siècle d'une lumière inédite " (Traverso, 2009). Cette ère des victimes, associée à «l'Ère du témoin» pour reprendre l'expression d'Annette Wieviorka (1998), s'accompagnent d'une métamorphose de la Shoah comme paradigme majeur de la mémoire occidentale. Précisons d'ailleurs qu'en termes de mémoires douloureuses à commémorer, I'Histoire, avec sa grande hache pour reprendre l'expression de Georges Perec (1975), n'a pas lésiné. Peu d'espaces géographiques échappent aux mémoires marquées par des violences diversement liées aux dictatures, aux crimes de masse, aux totalitarismes ou aux génocides. La reconnaissance de ces actes et des souffrances induites est aujourd'hui d'autant plus puissante que les flux migratoires produisent des diasporas planétaires en mal de mémoire (Appadurai, 2007) qui les inscrivent dans des interconnaissances mondialisées lesquelles alimentent les flux touristiques.

Les lieux ici analysés sont des lieux où des émotions - la terreur, la peur, l'épouvante - ont été à la fois produites et subies. Leurs mises en tourisme s'appuient sur les émotions qui y ont été vécues, pour les faire connaître et s'en souvenir, en fondant une relation lieuhistoire-mémoire accessibles par la visite, c'est-à-dire par ce qui re-donne vie au site par la présence et l'expérience des lieux. Les émotions constituent, ce faisant, la modalité humaine évidente, nécessaire et induite en même temps, pour rattacher un moment politique majeur d'inhumanité à un présent d'expériences individuelles et collectives. Ces lieux historiques sont devenus des sites, et les dispositifs spatiaux touristiques sur les emplacements de la Shoah sont rapidement devenus des références majeures pour l'édification de monuments dédiées à d'autres tragédies. Le principal vecteur de médiation mobilisé aujourd'hui par la mise en tourisme des lieux de mémoire douloureuse est celui de l'expérientiel qui vise à immerger le touriste dans un espace-temps où il expérimente, et éprouve le lieu, au double sens de ressentir et de mettre à l'épreuve. Le registre de l'émotion fait donc partie de l'actuelle panoplie professionnelle et opérationnelle de la mise en tourisme - les corps et les affects dès lors mobilisés dans et par la médiation entre des lieux et des publics - et les lieux de mémoire douloureuse n'y échappent pas. On pourrait même considérer qu'en la matière ils présentent des atouts propres pour la mise en marché touristique, puisque contrairement au Grand Canyon ou à la Mer de glace, ce sont des lieux que l'on visite justement parce que d'autres humains y ont vécu les émotions de la terreur. Ce faisant, la mise en tourisme des lieux de mémoire douloureuse n'est pas sans s'apparenter à l'engouement et aux pratiques du dark tourism.

\footnotetext{
${ }^{1}$ Ces deux qualificatifs renvoient aux visites effectuées sur les lieux de ghettos juifs.

${ }^{2} 500000$ visiteurs à Auschwitz en 2001, 1,3 million en 2009 et chiffre record, 1,5 million en 2014.
} 
Dans ce contexte, la marchandisation intrinsèque au tourisme (packaging des tours operateurs, coût d'entrée, produits dérivés) n'a pas manqué de soulever des controverses de nature éthique et morale. Sans débattre ici sur le fond de ce questionnement - peut-on moralement "vendre " des artefacts en lien avec ces mémoires traumatiques ? - on investiguera en revanche les modalités avec lesquelles les aménagements et les scénographies sont conçus pour dire et faire éprouver des émotions et ce qui s'y jouent, à titre individuel et collectif (Urbain, 2003). Après une mise en perspective des circulations mémorielles et des interactions émotionnelles qui se jouent dans ces lieux (in situ/ex situ), l'article analysera plus précisément les agencements muséographiques et les scénographies dans leur projet de co-mémoration et de transmission par le registre de l'émotion.

\section{Émotions et lieux de mémoires : circulations et interactions}

L'“ émotion ", promise par la communication des sites, est symétriquement attestée par les touristes après leur visite et certifiée comme réellement avérée par leurs témoignages dont les sites internet et livres d'or sont une première expression. Ainsi, les commentaires postés par les internautes sur le site Tripadvisor $^{3}$ constituent un matériau significatif, manifestant tout à la fois le désir de dire (ses impressions, ses émotions) et celui de partager des expériences complexes.

Bonne expérience de traverser les tunnels [de Cu Chi au Vietnam], il ne faut surtout pas être claustrophobe. Possibilité de tirer à I'AK47, M16... Je m'y suis laissé tenter malgré l'incohérence entre ce qu'il (sic) s'est passé ici et le fait de vouloir tirer à l'arme de guerre, je l'avoue... [...]. Visite incontournable. (27/08/2015).

[un] Moment émouvant [la visite d'Auschwitz], surtout que nous avions eu la chance d'être avec un rescapé. Le plus dur pour moi a été la pièce avec tout les restes des affaires personnelles des personnes massacrées: cheveux, lunettes, chaussures... (26/08/2015).

Les remarques, généralement empathiques - "Moment très émouvant ", "Expérience inoubliable ", "visite qui me marquera à jamais ", "incontournable ", "il suffit d'arriver pour sentir une drôle de sensation "- expriment des émotions, parfois sur un registre emphatique, qui ne diffèrent pas nécessairement des traces coutumières de l'impression touristique. Un internaute résume sa visite d'Auschwitz : "À vivre une fois dans sa vie ", à l'instar, finalement, du Grand Canyon ou la Grande muraille, comme si tous ces sites touristiques, pourtant bien différents, étaient comparables, et que les émotions fortes étaient la marque et la preuve en même temps de hauts lieux touristiques, quelle qu'en soit la teneur (terreur ou saisissement esthétique). Cette grandiloquence (Rosset, 1977) traduit la difficulté bien humaine de trouver l'expression juste pour signifier l'expérience d'un lieu et d'un moment, c'est-à-dire établir une relation entre des mots et des émotions, et ce, quel que soit le statut du lieu par rapport au événements traumatiques.

Il faut en effet, dans un premier temps, distinguer les lieux mêmes où ils se sont déroulés, plus ou moins préservés "en tant que tels", plus ou moins aménagés et plus ou moins sanctifiés, par les autorités et/ou par les visiteurs, et les lieux de commémoration

\footnotetext{
${ }^{3}$ Sauf mentions contraires, les extraits du site Tripadvisor proviennent d'une consultation effectuée le 28 août 2015.
} 
"délocalisés ", spécialement produits et aménagés pour évoquer et commémorer " au plus près " les tourments et lieux de souffrances. Cette distinction " d'aires des victimes " est importante: les scénographies in/ex situ se déclinent différemment - en fonction des contraintes de sites en particulier -, et mobilisent des registres particuliers de monstration : être la où / être dans un lieu différé, alors même que le sensible (ce qui passe par les sens) et l'émotion soient mobilisé dans les deux cas.

Le lieu historique est transformé pour recevoir des visiteurs. II n'est plus celui où se produisirent les atrocités. Ainsi, lorsque P. Levi retourne à Auschwitz en 1965, sa visite au Camp principal ne lui fait pas grande impression.

Le gouvernement polonais l'a transformé en une sorte de monument national; les baraques ont été nettoyées et repeintes, on a planté des arbres et dessiné des platesbandes. Il y a un musée où sont exposés de pitoyables vestiges: des tonnes de cheveux humains, des centaines de milliers de lunettes, des peignes, des blaireaux, des poupées, des chaussures d'enfants; mais cela reste un musée, quelque chose de figé, de réordonné, d'artificiel. Le camp tout entier m'a fait l'effet d'un musée (Lévi, 1987, Question 4 : 199)

Pour cet homme, emprisonné à Monowitz en 1944, qui a vécu l'expérience concentrationnaire, l'aménagement d'Auschwitz comme lieu de mémoire échoue dans ses objectifs. En revanche Birkenau, où aucune conservation, ni restauration n'ont été effectuées, ravive souvenirs traumatiques et émotions.

Là, rien n'a changé, il y avait de la boue, et il y en a encore... là rien n'a été enjolivé (Lévi, 1987, Question 4 : 199).

C'est que la transmutation du lieu d'épouvante et d'inhumanité en lieu de visite et de comémoration s'effectue à la fois par une médiation de l'ordre d'un processus politique et des interventions logistiques sur site qui en modifient l'agencement. La création du musée d'Auschwitz remonte à 1947; une loi stipule alors que le musée avait pour but de " commémorer le martyre de la nation polonaise et des autres nations " (Weizmann, 2005 : 87). Le "pavillon juif » ne verra le jour qu'en 1968. La concurrence des mémoires (Chaumont, 1997) se poursuit de plus belle avec l'installation de carmélites dans un ancien dépôt de boîtes de Zyklon B. Ce n'est qu'en 1992, sous la pression du Vatican, que les Carmélites acceptent finalement de déménager, à deux kilomètres du camp.

À Cracovie, les voyages clés en main proposés par de nombreux tours-opérateurs vers le symbole du mal absolu de la société occidentale contemporaine (Wieviorka, 2011) prolifèrent. L'aller-retour Cracovie-Auschwitz, en autocar, inclut une visite commentée par un guide du musée. Le trajet constitue d'ailleurs un espace-temps particulier, pendant lequel les touristes se préparent à cette visite. Pour celles et ceux qui savent, les visages se ferment à mesure que le plus grand cimetière du monde approche ; les forêts de bouleaux constituent déjà un premier marqueur spatial du drame qui s'est déroulé en ces lieux. Mais, pour la majorité, la visite du camp d'Auschwitz commence véritablement sous le fameux portail "Arbeit macht frei » (Figure 1), dont la traduction s'avère sans doute tout à fait inutile. À cet endroit les appareils photographiques sortent des sacs et les guides commencent leur travail d'accompagnateur. Dérobé en 2009 et scindé en trois morceaux pour le compte d'un néo-nazi suédois, ce fronton, ressoudé en 2011, a désormais retrouvé sa (sinistre) place et représente le seuil d'un monde vers un autre. 
Figure 1 : Entrée d'Auschwitz avec son fameux portail « Arbeit macht frei »

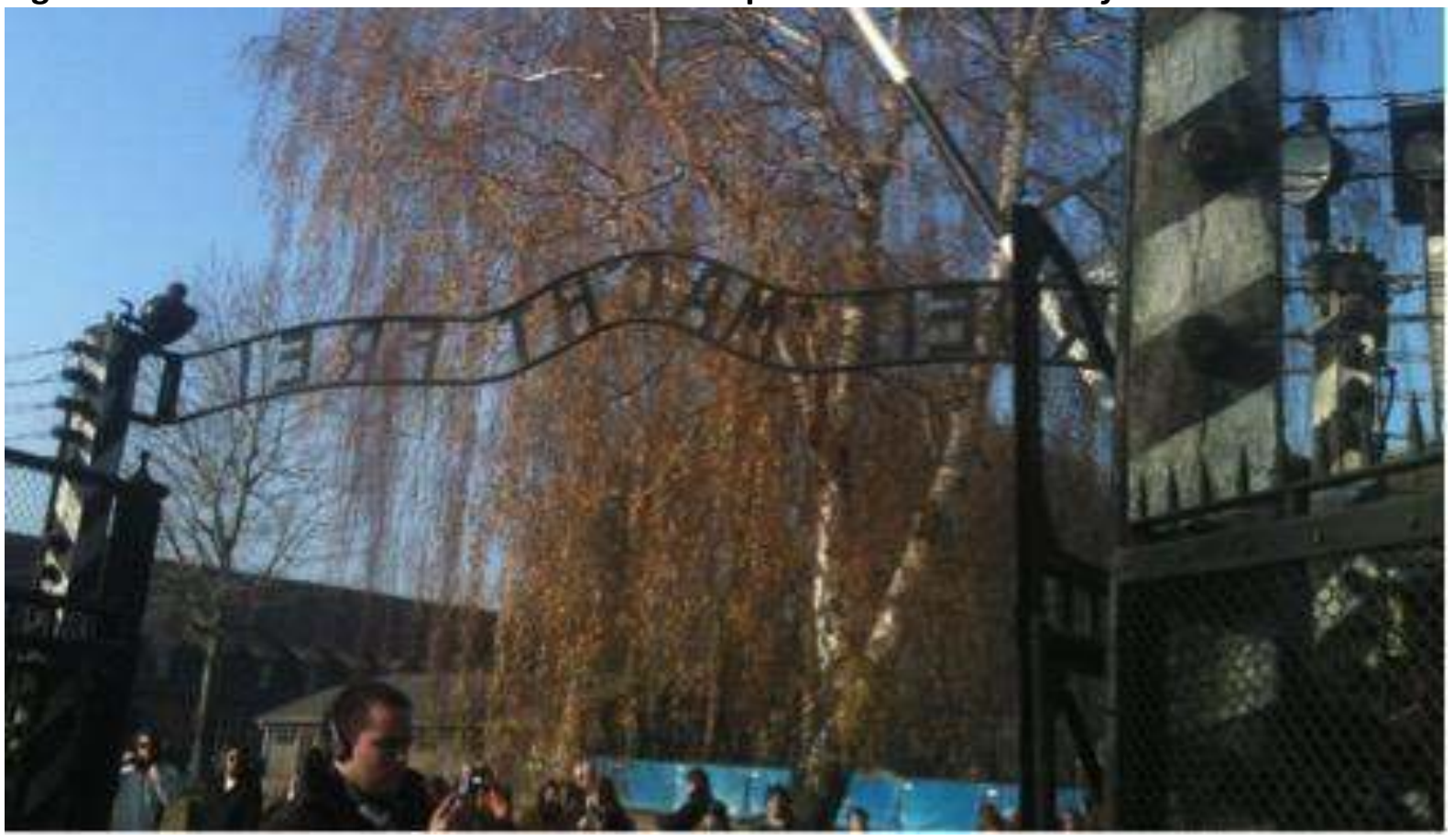

Cliché : Dominique Chevalier, novembre 2011.

Le voyage individuel traumatique de la visite et la confrontation existentielle de l'individu face à la/sa mort combinent, sur le lieu, rencontre avec un lieu historique et production de mémoire. Mais ces lieux, comme d'autres, ne sont pas à l'identique de leur réalité passée. Ils ont été modifiés et leur adaptation à la visite touristique se traduit par de nombreux aménagements.

Un processus de cosmétisation, par enjolivement mais surtout peut-être par ordonnancement, construit à la fois la possibilité même de la visite et sa dénaturation. Un lieu mis en ordre et des traces classées pour devenir des collections sont autant de signes d'une relecture archivistique et historienne d'un crime de lèse-humanité. La mise en ordre, c'est-à-dire la mise en sens, effectue intrinsèquement une re-construction de signification de ce qui touche à l'inhumanité, c'est-à-dire à l'in-sensé. On comprend dès lors mieux pourquoi et comment le registre émotionnel est convoqué, que l'on table sur l'émotion spontanée et induite par la simple présence aux emplacements des mises à mort, ou que l'on mette en œuvre, volontairement, des scénographies émotionnelles. L'émotion constitue la médiation immédiate entre humains, le medium partagé et partageable, nécessaire à la connaissance, par les sens, de ce qui n'en a pas. L'émotion, dès lors, constitue simultanément un indicateur d'authenticité du lieu (même si celui-ci ne ressemble plus guère à ce qu'il fût) et un opérateur de conscience, c'est-à-dire un ressort compréhensif et de possible intellection historique.

L'Histoire ici est submergée de mémoire(s) en construction (Nora, 1984), en élaboration continuée par la pratique même de la visite. Les touristes participent, par leur présence même, de la fabrication d'un récit qui transmute, toujours et nécessairement la première (l'histoire) en une seconde (la mémoire), changeante parce que continûment historicisée (Ricoeur, 2000). La visite donne vie au site et les touristes, alors témoins, convertissent leur présence vivante en témoignage de mémoire. 
En revanche, pour les musées, lieux différés (délocalisés) de la mémoire douloureuse, le registre émotionnel résulte essentiellement de scénographies où espaces de déambulation ouverte ou fermée, lieux d'exposition, reconstitutions et espaces intermédiaires, présence d'œuvre d'art, etc., constituent des dispositifs spatiaux, au service d'un projet muséographique revendiqué comme éducatif et attractif.

\section{Figure 2 : Guide du musée juif de Berlin distribué au musée}

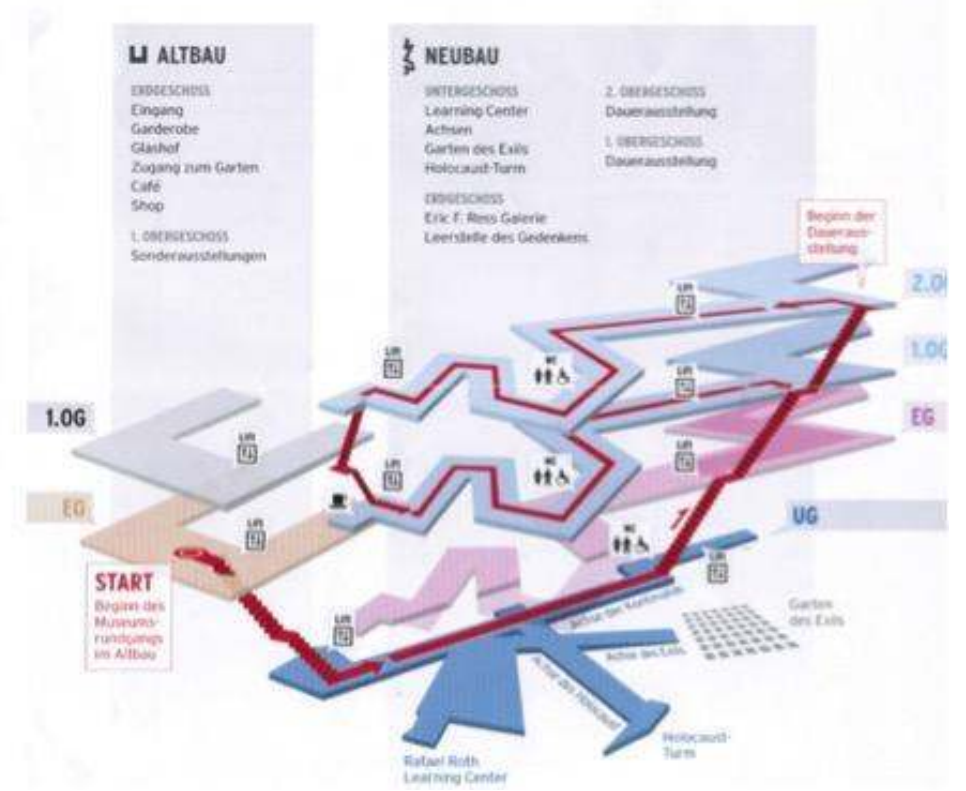

Source : Musée juif de Berlin.

La muséographie du musée juif de Berlin (Figure 2) illustre la performativité du discours et du geste architectural, destinés à produire ce qu'ils énoncent. Le parcours rouge correspond à une visite chronologique, sur quatre niveaux, de l'apport des Juifs à la société allemande depuis 950 ap. J.-C. jusqu'à aujourd'hui. Deux autres axes (bleus sur le guide) correspondent I'un à l'annihilation qui se termine avec la tour de l'Holocauste, l'autre à l'axe de l'exil qui débouche sur le Jardin de l'Exil. Les personnes qui empruntent l'axe de l'Holocauste entendent, comme au Musée de la Tolérance de Los Angeles, une lourde porte se refermer. L'objectif d'un " espace vide et menaçant " de l'architecte Daniel Libeskind (2005) est confirmé. Le malaise est également palpable dans le Jardin de l'Exil. Intentionnellement mouvant, le sol provoque un sentiment de déstabilisation pour qui le foule, dans le but de figurer les désorientations de toute personne exilée contrainte de vivre dans un univers qui n'a pas été choisi. Ces trois axes correspondent finalement à la réalité des Juifs allemands (ceux qui ont réussi à se cacher, ceux qui ont été assassinés, et ceux qui se sont exilés). Le centre éducatif constitue un quatrième axe, en tant que lieu d'éducation des jeunes générations ${ }^{4}$.

Dans les musées coexistent, en synergie, collections et scénographies artistiques dont la fonction est de transmettre en sensibilité et émotions (effroi, empathie, recueillement, culpabilité, fraternité...). Cette esthétisation, à la fois aesthésis (expérience sensorielle) et

\footnotetext{
${ }^{4}$ Ce centre éducatif n'est pas spécifiquement présenté comme un axe. Pourtant, cet objectif constitue une des missions essentielles du musée.
} 
expression artistique, vise une "incorporation » du lieu par les visiteurs. Les émotions sont évidemment ressenties avec intensité, mais elles sont induites tout à la fois par les objets, la respiration spatiale du dispositif muséal, et par les œuvres d'art présentes dans le dispositif. La cosmétisation des lieux est à la fois partie prenante de scénographie et de production de connaissances (présentations d'archives). Ainsi, au sein du parcours du « Museum of Jewish Heritage, A Living Memorial to the Holocaust » de New York, un jardin de pierres conçu par Andy Goldsworthy à la fois comme lieu de mémoire et lieu de méditation (Figure 3), permet conjointement de se reposer, de reprendre haleine et d'admirer le panorama d'Ellis Island et sa fameuse statue de la Liberté. L'artiste, figure majeure du Land Art, y a imaginé un jardin composé de dix-huit ${ }^{5}$ blocs de pierre, au sein desquels s'élève un chêne nain. L'ensemble, en donnant toute sa place au "vide ", celui de l'absence, ce vide interstitiel nécessaire pour attester d'un plein, aujourd'hui pétrifié, symbolise ainsi l'enracinement de l'instinct de survie, autant qu'il joue un salutaire rôle de sas pour méditer, et absorber les traumatismes entrevus et les émotions ressenties.

\section{Figure 3 : Jardin de Pierres, Museum of Jewish Heritage, New York}

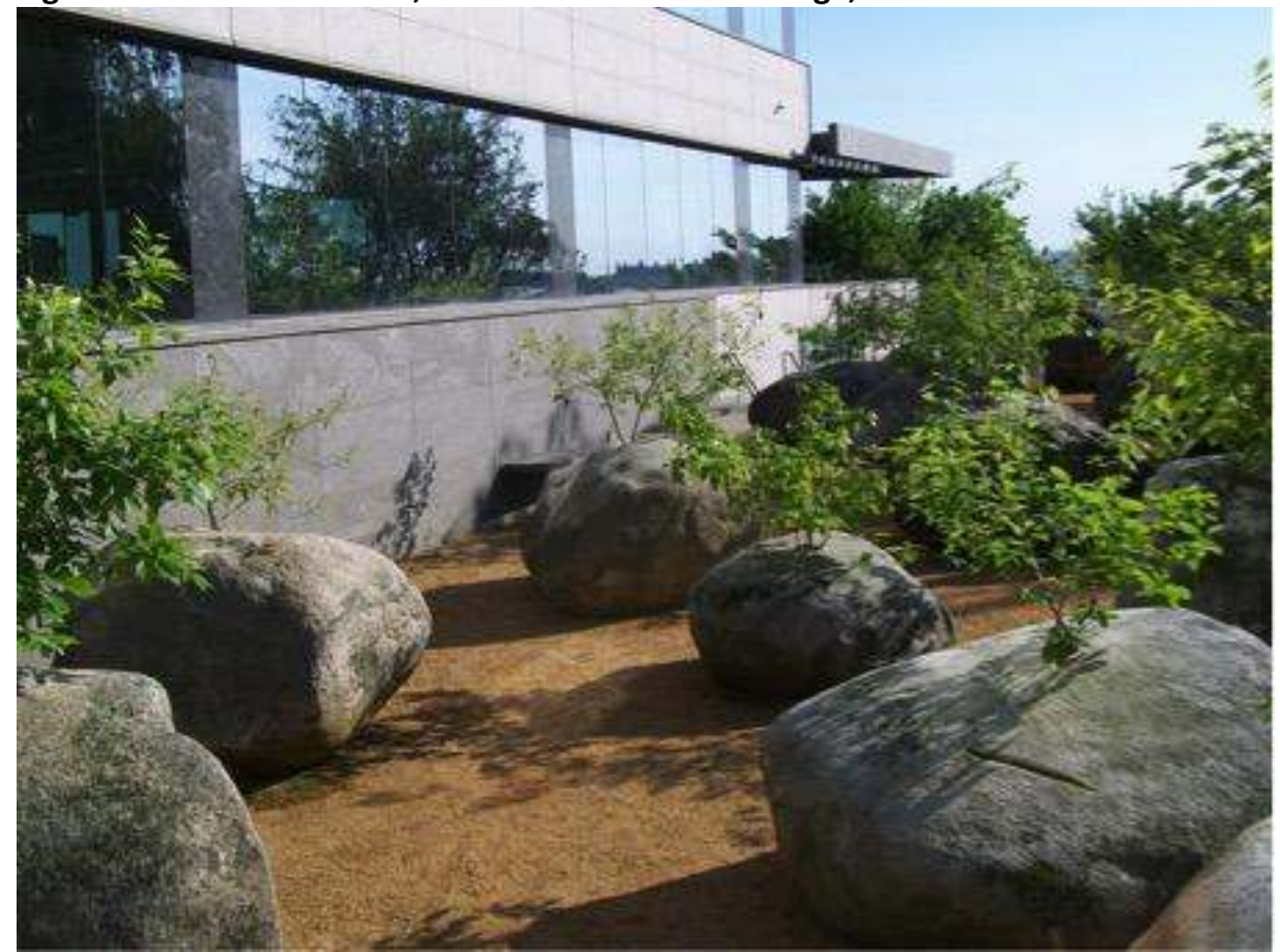

Cliché : Dominique Chevalier, janvier 2008.

Enfin, comme vecteur privilégié de médiation, le principe d'incorporation donne lieu, au sens le plus littéral des termes, à de véritables expériences physiques in situ. Telle semble être la teneur des propos laissés sur le site Tripadvisor par les visiteurs des tunnels de Cu Chi, au Vietnam.

Situés à une cinquantaine de kilomètres au nord de Hô Chi Minh-Ville (ex Saigon), ces tunnels constituent une partie du complexe militaire de Ben Dinh. Devenus sites

\footnotetext{
${ }^{5}$ La valeur numérologique 18 du mot hébreu chaï signifie vie.
} 
touristiques reconnus, inscrits au programme de nombreux tours organisés, ils symbolisent à eux seuls la résistance vietnamienne et la guerre contre les Américains (Giblin, 2007). Ces " villes souterraines " dissimulaient, sur plus de deux cents kilomètres, des cachettes de 60 à 70 centimètres de large, sur une hauteur de 80 à 90 centimètres. "Comment ont-il fait pour survivre la dessous ? » se demande un visiteur dans un commentaire laissé le 27 août 2015. Agent orange, napalm, bombardements classiques et perfectionnés ont échoué à déloger les soldats vietnamiens du Viêt Cong de ces inconfortables cachettes souterraines. Cette combativité, basée notamment sur l'inventivité et l'ingéniosité, est promue avec fierté par les guides qui accompagnent les touristes et par les reconstitutions de scènes de la vie quotidienne répartis le long du parcours : trappes camouflées avec soin, faux sols pivotants aboutissant sur des rangées de bambous finement acérés pour piéger l'ennemi, fumée des cuisines s'échappant à plusieurs centaines de mètres, empreintes de chaussures inversées... En début de visite, de nombreux touristes, curieux de vivre cette expérience de vulnérabilité, testent l'entrée dans les tunnels; pour l'album de photographies familiales, ils n'hésitent pas à mettre de jeunes enfants sur leurs épaules. 
Figure 4 : Expérimentation des tunnels de $\mathrm{Cu}$ Chi par les touristes
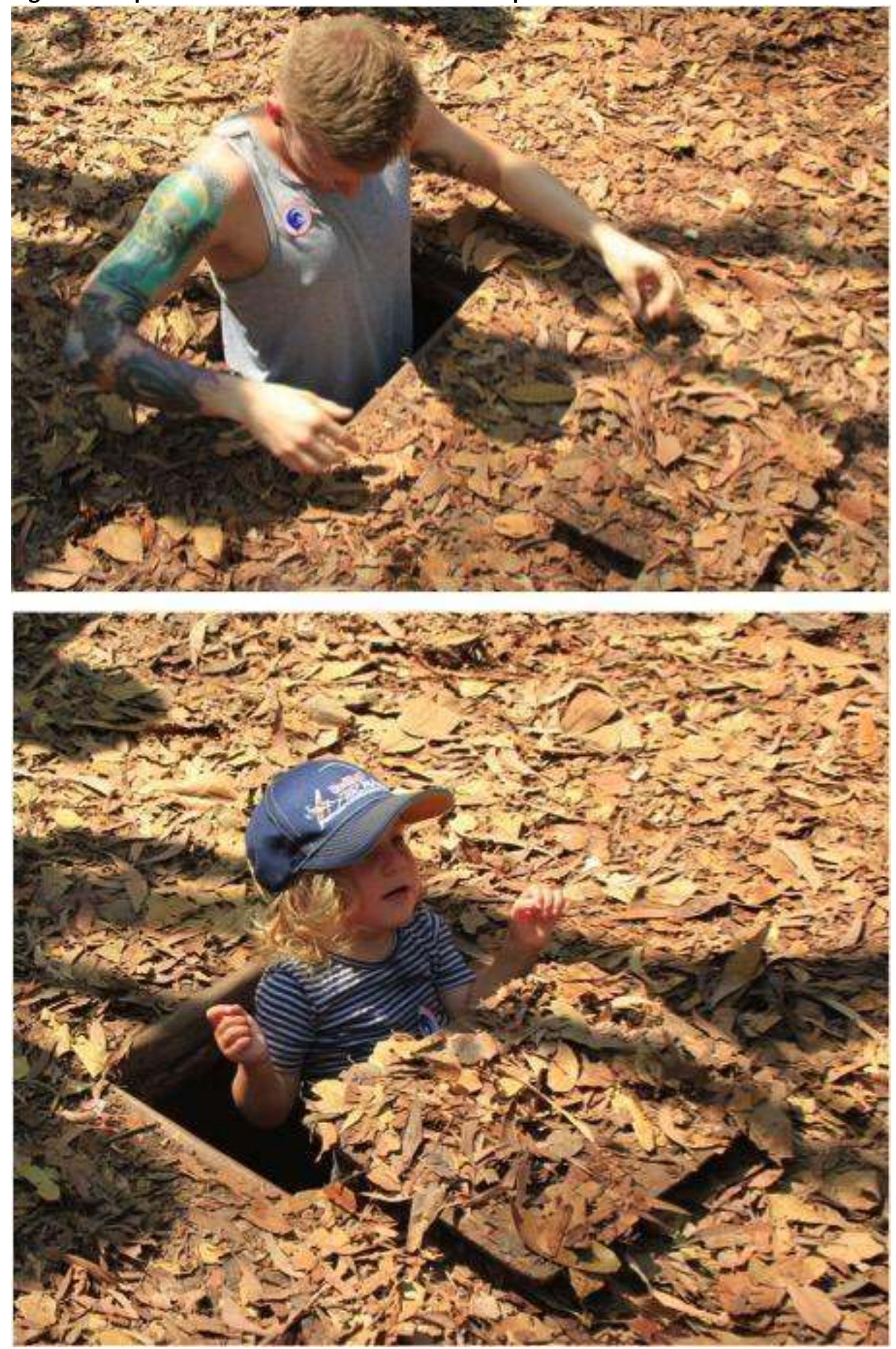

Cliché : Dominique Chevalier, avril 2015. 
À mi-parcours, une portion de tunnel ouverte sur une section de cent mètres fournit aux touristes la possibilité d'en faire l'expérience (Figure 4). Éclairés - et élargis par rapport aux originaux, les Occidentaux étant généralement de stature plus forte que les Vietnamiens -, les touristes progressent dans le tunnel, courbés dans les entrailles de cette terre généreusement arrosée de napalm et d'agent orange. Pendant le conflit, ce n'est pas en se déplaçant accroupis ou fléchis mais en rampant parmi les rats, les fourmis et autres insectes, dans la puanteur et l'obscurité que les Vietnamiens se déplaçaient. "Durant la saison des pluies, [le sol] était souple comme du sucre et pendant la saison sèche il était dur comme la pierre $\|^{6}$. Au plus fort du conflit, les tunnels hébergeaient les vivants et les morts, recouverts de glaise et placés en position fœtale, en attente d'une sépulture décente. Quelques corps de soldats américains ont connu le même sort, afin de démoraliser leurs camarades, déprimés par la perspective de croupir ensevelis et de ne pas avoir de funérailles décentes au pays. Aujourd'hui, tous les vingt mètres, une sortie permet de s'extraire du tunnel, en cas de panique.

Paradoxalement, la douleur, les souffrances et les privations endurées par les Vietnamiens ne sont pas clairement explicitées. Les commentaires insistent en revanche sur l'ingéniosité et l'abnégation des résistants. Les épreuves sont présentées en filigrane, et finalement les souffrances endurées par les Américains ${ }^{7}$ sont davantage mises en avant, comme châtiment mérité (Figure 5).

Ces sites, jouissant actuellement d'un tourisme domestique vietnamien en pleine expansion, servent (à) l'élaboration et la démonstration d'un discours politique au service d'une nation " unie et unanimement socialiste » (Cabasset-Sebedo et al., 2010 : 234). Les sites de guerre, et tout particulièrement les tunnels de $\mathrm{Cu} C h i$ constituent de fait des instruments spatiaux de choix pour exprimer la souveraineté de la nation. Dans ce contexte, les touristes occidentaux peinent à trouver leur place. S'ils sont généralement familiarisés avec les horreurs de la guerre du Vietnam par le biais de films célèbres (Good Morning Vietnam, Full Metal Jacket, Forest Gump, Né un 4 juillet, etc.), leur empathie oscille. Qui sont les victimes désignées dans ce lieu, et donc avec quelles victimes s'identifier? Beaucoup sont manifestement troublés, peut-être en raison des douloureux souvenirs liés aux tranchées de la Première Guerre mondiale ? En tout cas, s'il n'est pas envisageable de nier les tourments et souffrances endurés par le peuple vietnamien, il reste néanmoins difficile, pour beaucoup d'Américains, d'Australiens ou d'Européens d'apprécier à leur juste valeur les subtilités des pièges dans lesquels les Américains tombaient.

\footnotetext{
${ }^{6}$ Vien Phuong, poète et écrivain saïgonnais qui a passé la plus grande partie de la guerre dans les tunnels, in Mangold, T. et Penycate, J. "Les rats du tunnel ", Spécial NAM, L'histoire vécue de la guerre du Viet-Nam, $\mathrm{N}^{\circ} 28,1985$.

${ }^{7}$ Pour trouver traces des horreurs de la guerre côté vietnamien, c'est au musée des vestiges de la guerre qu'il faut se rendre, où le premier étage est notamment consacré aux effets et aux conséquences de l'utilisation de l'Agent Orange sur la population et sur les espaces, et aux agressions et atrocités commises par les Américains. Notons par ailleurs que la question des Boat People n'est jamais abordée.
} 
Figure 5 : Démonstration du fonctionnement des pièges et illustrations peintes sur les murs
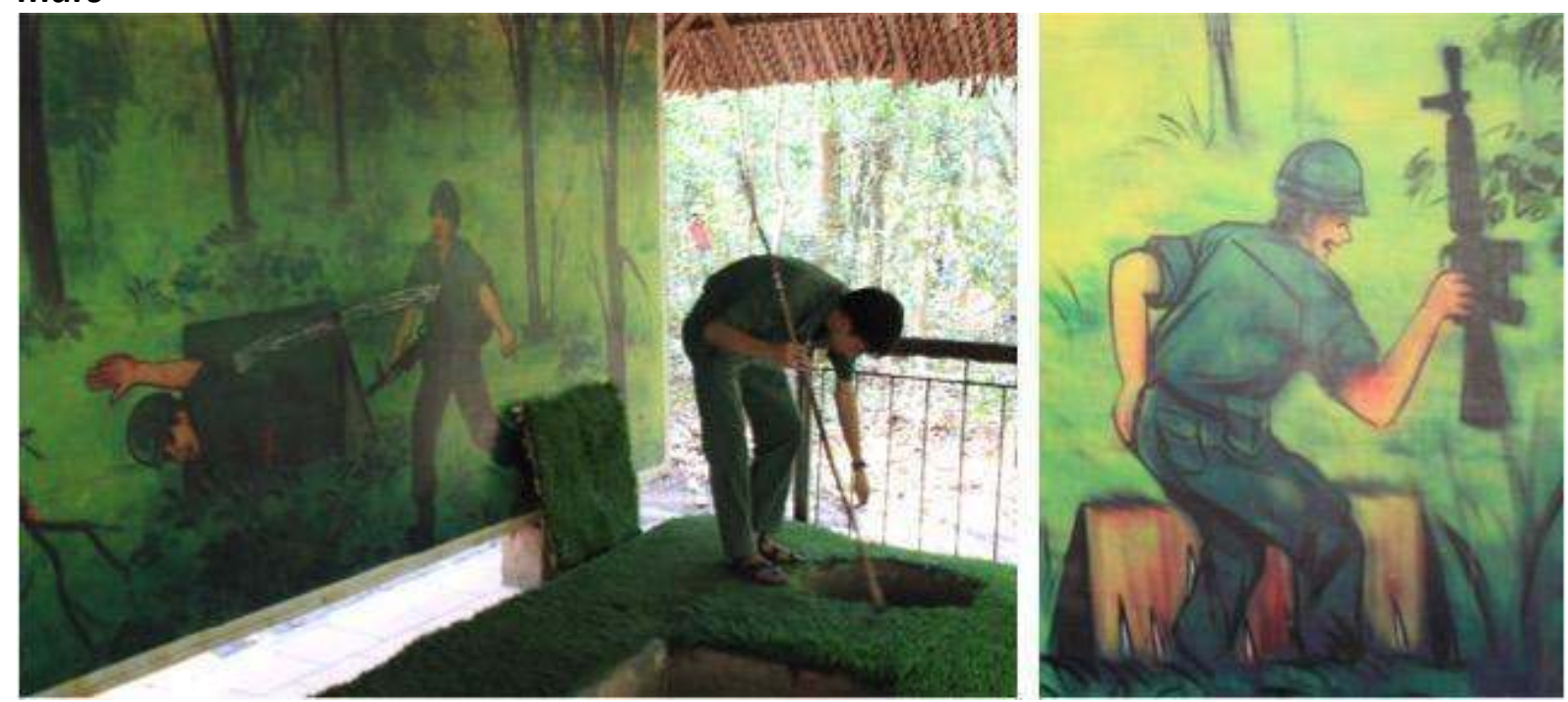

Cliché : Dominique Chevalier, avril 2015.

L'émotion n'est plus ici induite (simplement) par la vue, mais par l'expérience propre d'un corps qui éprouve un espace. Le visiteur n'est plus seulement spectateur mais spect-acteur, selon les principes contemporains de la médiation. Ce faisant, le faire avec l'espace est mobilisé, de façon pédagogique, pour induire, par l'expérience corporelle multisensorielle (l'odeur, le toucher, la vue) une re-présentation en acte de ce que fut celle des populations que l'on vient commémorer.

Dans les deux dispositifs touristiques et de visites, in/ex situ, les modes opératoires participent d'une semblable " expérience esthétique comme expérience d'un sens partagé » (Caune, 1999). Mais l'authenticité historique du lieu - où s'est effectivement produit l'événement - n'est pas corrélée à une " authenticité » émotionnelle qui lui serait propre et le distinguerait des lieux ex situ. En effet, les pratiques qui s'y manifestent ont montré ${ }^{8}$ combien l'expérience de ces espaces provoquait des émotions individuelles et/ou collectives de grande intensité, comparables voire parfois plus visibles à celles exprimées dans les lieux historiques. À Auschwitz le poids du lieu, mettre ses pas dans les pas des victimes, a/terre, alors qu'à Washington, Los Angeles, etc. - parce qu'elle n'est qu'idéelle -, la présence des centres de mise à mort libère l'expression des émotions. In fine, la différence entre ces deux types de lieu s'amenuise dans des expériences émotionnelles qui les confondent dans une même mémoration. Le propre géographique du lieu se trouve dès lors dépassé par ses représentations mêmes, mentales, idéelles et matérielles. Ce n'est pas le lieu de mémoire (in situ), mais la/les mémoire(s) des événements, qui en circulant collectivement, suscitent les émotions.

L'aménagement de ces lieux n'obéit pas seulement à une volonté politique et éthique de rendre compte d'un savoir historique sur le passé. II répond également aux impératifs besoins de se souvenir et de commémorer des sociétés contemporaines, pour fabriquer de

\footnotetext{
${ }^{8}$ Ces constats reposent sur des observations effectuées sur les différents terrains mobilisés dans cet article. L'objet même de ces questions et des lieux rend délicat un travail d'enquêtes formalisées, et il a été préféré des approches empiriques et d'observation. On touche, à cet endroit, la question méthodologique et réflexive du chercheur et sans doute aussi les limites de l'investigation scientifique dans le domaine de l'émotion.
} 
la mémoire et une identification à un collectif. La qualification de ce dernier soulève évidemment le problème de sa référence : celle d'une " Humanité " qui transcende toutes les autres catégories d'identité ou celle d'un groupe particulier, ici national, ailleurs ethnique. C'est que la mémoire douloureuse, toujours celle de groupes singuliers, dit à la fois I'Un de l'Humain et le pluriel des appartenances. En conjoignant (expérience) mémorable et mémorisable, la présence touristique traduit/transfert le champ de l'émotion à celui du politique, pour susciter, induire et inculquer un sens, c'est-à-dire un discours, inscrit à l'intérieur de frontières (ethniques ou nationales) ou les transcendant toutes. Cette équivocité même ne plaide en rien pour une efficience avérée du projet de la mémoration et de la visite.

\section{L'émotion, jusqu'où ?}

Un avis posté sur Tripadvisor (12 août 2015) critique à la fois l'approche du musée d'Auschwitz, "pas suffisamment connecté au monde actuel contrairement au musée d'Hiroshima " et le comportement des touristes jugé "scandaleux ": " ce n'est pas une attraction touristique mais un lieu de mémoire " précise cet internaute qui souhaiterait, par ailleurs, interdire de photographier à l'intérieur des camps.

Entre empathie, sympathie, compassion ou indignation, les remarques de ce type interrogent $\mathrm{I}^{\prime}$ (in)adéquation des émotions et leur réception, en fonction des lieux, des publics et du souvenir des victimes. Comment faire avec ses/ces émotions ? L'absence d'émotions ou de manifestations d'émotions est d'ailleurs tout aussi équivoque qu'un embarrassant excès inverse, les émotions pouvant par ailleurs être contagieuses. Il existe, de manière implicite, une gamme d'expressions émotionnelles acceptables pour le collectif et soumises à une véritable discipline sociale (Halbwachs, 1947). Finalement, incorporée dans divers agencements et confrontée à différentes expositions d'archives et de témoignages, qu'est-ce qu'une manifestation d'émotion collectivement, moralement et socialement acceptable ? La société façonne les sensibilités aussi sûrement qu'elle modèle les cadres du langage et de la pensée collective.

II nous vient alors en mémoire le souvenir de deux adolescents, plutôt turbulents, rencontrés lors d'une visite du musée de l'Holocauste de Montréal. Nous les avions suivis le temps de cette visite qui s'inscrivait dans le cadre d'une sortie scolaire. Ils semblaient agités et contenaient avec peine des fous rires. Sans doute par confort, au cours de leur déambulation, ils choisirent de s'asseoir devant un écran où une rescapée, au crépuscule de sa vie, racontait avec émotion son enfance marquée par la déportation et l'expérience d'Auschwitz. D'abord satisfaits d'avoir trouvé une place assise, les deux adolescents ne montraient pas, a priori, un grand intérêt pour le récit de cette dame. Puis l'évocation du quotidien, du froid, de la faim, tenace, et de la mort les a interpellés. Leurs yeux se sont détachés de l'écran pour se regarder l'un l'autre; dans cet échange de regards, ils ont exprimé rapidement mais avec insistance une petite moue avec leur bouche, ont écarquillé les yeux, puis assurés que l'autre partageait le même état de stupéfaction, ils ont poursuivi leur écoute du témoignage, et terminé la visite avec un comportement " conforme au lieu » et sans "fausses notes ».

Précisément, cette question de la décence du comportement revient dans de nombreux commentaires de visiteurs de lieux de mémoires douloureuses. Qu'est-ce qu'un 
comportement décent, acceptable, convenable dans un lieu de souffrances extrêmes ? Peuton se promener en short, boire et manger à Auschwitz? "À partir de quand peut-on recommencer à rire ? " demandent d'ailleurs certains élèves à la suite de leur visite. Peut-on prendre des photographies ? Se prendre en photographies ? Faire des selfies devant un four crématoire, devant la rampe de sélection, devant le fameux panneau " Arbeit Macht Frei »? "Should Auschwitz Be a Site for Selfies ? " s'interroge Ruth Margalit dans le New Yorker du 26 juin 2014. Quelques jours auparavant, la publication sur Twitter d'un selfie d'une adolescente américaine avait embrasé les réseaux sociaux, au point que la jeune fille (Figure 6) reçut plusieurs menaces de mort et plus de 6000 réponses haineuses en 24 heures. Semblablement, en 2010, "Adolek » Kohn, un Juif polonais de 89 ans, survivant d'Auschwitz et réfugié en Australie en 1949 avait créé une polémique similaire. Sa fille, une artiste australienne, l'avait filmé en train de danser à Auschwitz avec ses petits-enfants, sur I'air de I Will Survive de Gloria Gaynor. Elle avait ensuite posté sur Youtube cette performance. Sous le poids des critiques, cette vidéo a ensuite été retirée 9 .

\footnotetext{
${ }^{9}$ http://www.slate.fr/lien/26167/dancing-auschwitz
} 
Figure 6 : Selfie posté sur les réseaux sociaux

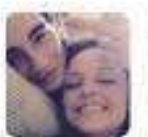

Princess Breanna

ig PrincessBMM

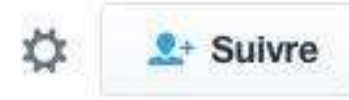

\section{Selfie in the Auschwitz Concentration Camp}

6 Voir la traduction

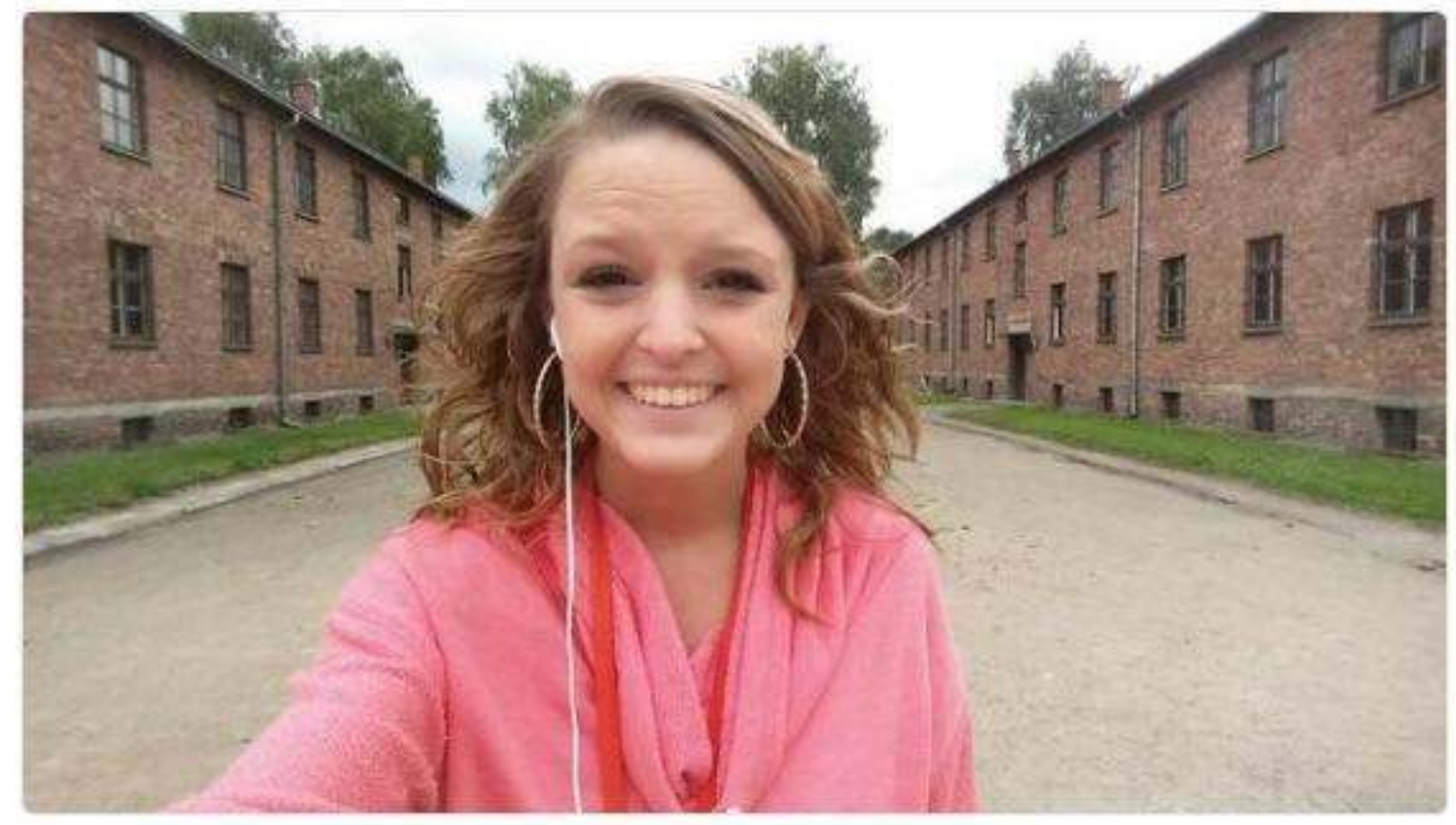

RETWEETS JAIME

$18709 \quad 17375$

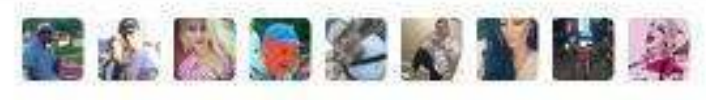

$13: 11$ - 20 juin 2014

Source : Compte Twitter @PrincessBMM, 20 juin 2014, en ligne: https://twitter.com/PrincessBMM/status/479944652049895424

Plus récemment encore une polémique ${ }^{10}$, liée à l'installation de douches rafraîchissantes à l'entrée du camp (Figure 7), montre combien, in situ, la prise en compte du rapport à l'espace et au temps varie et influence différemment le fonctionnement social de la mémoire et de ses lieux. En août 2015, un visiteur israélien ayant perdu une partie de sa famille pendant la Shoah, choqué par cette nouvelle installation, a informé les autorités du mémorial de sa consternation. Si ce malaise a souvent été partagé, y compris dans la presse et les réseaux sociaux, plusieurs groupes n'ont manifestement pas fait le parallèle entre cette installation et les "douches " où les Juifs étaient conduits pour y être gazés. La mise en place de ces douches/brumisateurs a d'ailleurs parfois donné lieu à de joyeux éclaboussements. L'émotion a été particulièrement manifeste chez les touristes israéliens,

10 http://www.huffingtonpost.fr/2015/08/31/auschwitz-douches-visiteurspolemique n 8066392.html?ncid=fcbklnkfrhpmg00000001 
notamment les plus âgés ${ }^{11}$. Leur émoi était sans doute double. D'une part, le parallèle possible entre douches/brumisateurs et chambres à gaz, en ce lieu, a pu les offenser et, d'autre part, le fait que de jeunes Israéliens puissent s'amuser ainsi, dans le plus grand cénotaphe juif du monde, symbole de souffrances, traumatisme et barbarie, a pu leur sembler une attitude offensante, proche d'une profanation de la mémoire des leurs.

Figure 7 : Brumisateurs/douches installés à Auschwitz

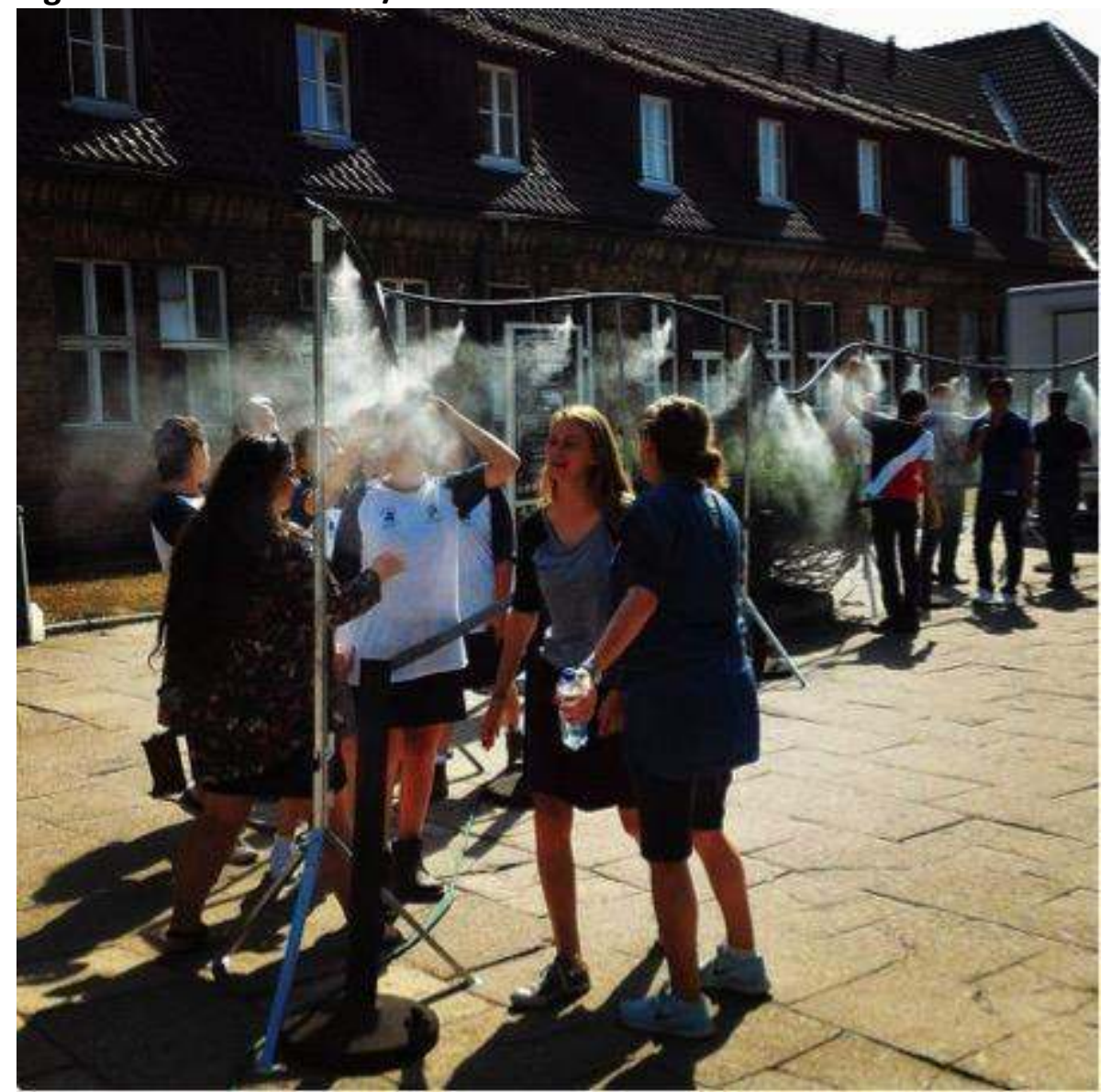

Source : imgur, via Le Figaro.fr, 01-09-2015.

Un porte-parole du musée d'Auschwitz a immédiatement réfuté le rapprochement douchesbrumisateurs/douches, en objectant que le Zyklon B, gaz mortel, " était diffusé de manière totalement différente " et que le plus important, en l'occurrence, était de minimiser les risques liés aux fortes chaleurs ${ }^{12}$. Justification froide et technique en situation caniculaire. $\mathrm{Ce}$ conflit d'acteurs et donc d'interprétations, exprime l'équivocité intrinsèque des lieux, et sans doute plus avant même l'hybridation spatiale de l'histoire et de la mémoire. Pour le dire autrement, l'hybridation problématique que les usages des lieux (passés et présents) induit, entre événements historiques et nécessités mémorielles, traduit l'irréductible relation entre des lieux et des valeurs (événementielle, mémorielle, économique).

Grâce au développement des compagnies aériennes low cost et à l'entrée de la Pologne dans l'Union européenne, le pays représente la $18^{\mathrm{e}}$ destination touristique mondiale en termes d'arrivées (2013) selon les données de l'Organisation Mondiale du Tourisme (OMT).

\footnotetext{
${ }^{11} \mathrm{http}: / /$ www.ynetnews.com/articles/0,7340,L-4696040,00.html

${ }^{12}$ http://mashable.com/2015/08/31/auschwitz-cooling-showers-offend/?utm cid=mash-com-Tw-main-link
} 
Souvent qualifiée de "joyaux d'Europe centrale ", Cracovie est rapidement devenue une destination prisée ; près de 9 millions de touristes l'ont visitée en 2012 et, selon le magazine en ligne Challenge, Cracovie serait la destination européenne la plus appréciée des touristes $^{13}$. Près de 200 tours opérateurs proposent des voyages aller-retour en bus avec visite dans les deux haut-lieux polonais incontournables, tous deux classés au patrimoine mondial de l'UNESCO : Auschwitz-Birkenau et la mine de sel de Wieliczka (Figure 8).

Figure 8 : Deux haut-lieux touristiques polonais incontournables

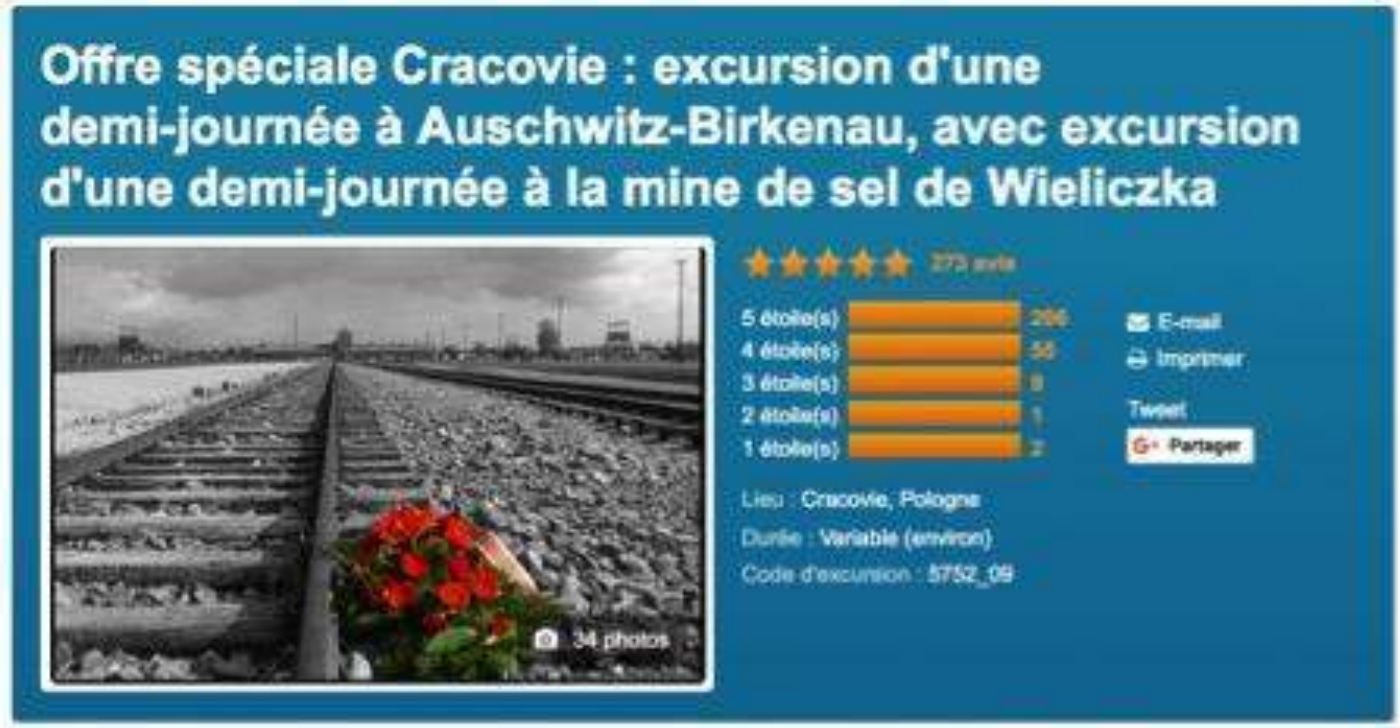

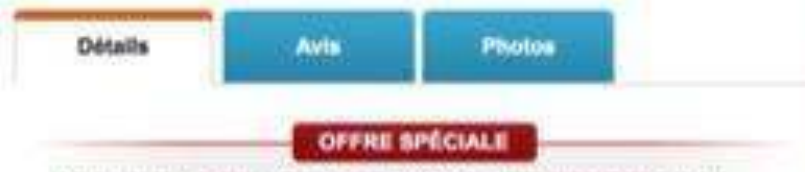

Rbeervoz et bonomisez jusqu'a $10 \%$ par rapport aux doux vithes achetbes separbment I

Visitez deux des endroits les plus popisaires de Pologne grace a cette offre spociale Cracovie qui combine doux excursions tres apprbciées sur deux jours a prix réduit t Le 1er jour, explorez run des plus tristement cébbres sites d'Europe, le complexe du carno de concentration ofAuschwilz-Birkengu, o0 vous decourirez quefques-unes des baraques de la prieon et en apprendrez plus sur les prisonniers dans des expositions spbcialos. Puis, te iendemain, partez scus la terre dans la mine de sel de Wieliczka et imerveillez-vous devant les formations de sel, telles que la superbe chapelle de St Kinga. Cette excursion est le moyen ibeal de decouvrir deux dos sites de Pologhe classbs au patrimoine mondial de RUNESCO.

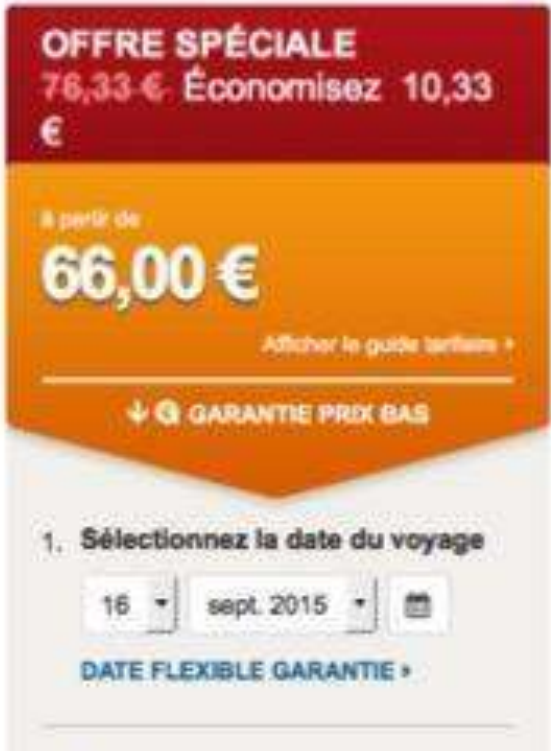

2. Indiquez le nombre total de

Source : Copie d'écran : http://www.viatorcom.fr/fr/7379/tours/Cracovie/Offre-speciale-Cracovieexcursion-dune-demi-journee-a-Auschwitz-Birkenau-avec-excursion-dune-demi-journee-a-la-mine-de-sel-deWieliczka/d529-5752 09

Ces questionnements ne sont pas propres à Auschwitz. De la même manière, il est sans doute légitime de se demander s'il est décent de tirer au fusil d'assaut à $\mathrm{Cu}$ Chi. Où

13

http://www.challenges.fr/economie/20140124.CHA9646/tourisme-cracovie-ville-d-europe-la-plusappreciee.html, page consultée le 13 septembre 2015. 
commence et dans quoi se niche cette impression d'indécence, cet écart de la décence aux lieux: dans une absence ou dans un trop plein d'émotions, dans des manifestations d'insensibilité ou dans des formes, diverses, de fascination ?

Ces questionnements éthiques et moraux ne concernent pas uniquement les lieux historiques. Situé très loin des centres de mise à mort, le parti pris architectural du musée de I'Holocauste de Washington (United States Holocaust Memorial Museum) tente de reconstituer, en plein cœur de la capitale fédérale, l'atmosphère des camps, à l'extérieur comme à l'intérieur du musée. L'architecte James Ingo Freed ${ }^{14}$, réfugié de l'Allemagne nazie à la fin des années 1930, a voulu et conçu une construction oppressante qu'il qualifie luimême de "viscérale ": I'utilisation de la brique rouge rappelle symboliquement les camps nazis, les escaliers extérieurs métalliques et les ponts suspendus le ghetto de Varsovie. Ainsi, les touristes qui visiteraient ce musée un peu "par hasard ", compte tenu de sa proximité, sur le Mall, avec les autres grands mémoriaux et musées nationaux, sont immédiatement placés dans le contexte oppressant de l'Holocauste ${ }^{15}$. En mettant l'accent sur l'ouverture des camps et l'accueil des réfugiés juifs après guerre, l'approche américaine de la mémoire de la Shoah se fonde sur une glorification et une forme d'auto-idéalisation de l'Amérique (Young, 1991). Finalement, la Shoah fournit un détour pédagogique de choix pour expliquer aux plus jeunes comment se comporter dans la société en général et dans la société américaine en particulier. Mais, si loin des centres de mise à mort, quel degré d'horreur était-il opportun de montrer $^{16}$ ? En tension entre d'une part exposer la réalité, cruelle, tragique et insoutenable et, d'autre part, enseigner, transmettre et commémorer - tout en rappelant les ambitions civiques américaines -, cette conception muséale exemplifie le défi d'un discours mémoriel à double finalité, celui de l'Humanité, celui de la Nation. Nombreux en furent les détracteurs qui ne manquèrent pas de souligner que ces discours détournaient l'attention du public d'enjeux mémoriels plus strictement états-uniens, tels que ceux des Natives et des Afro-Américains.

Des problèmes techniques muséographiques, confrontés à des questions d'ordre éthique, ont rapidement émergé. Ainsi, le transfert d'objets témoins/témoignages de leur lieu historique vers un autre, distant de plusieurs milliers de kilomètres, pouvait-il se justifier d'un point de vue déontologique ? Fallait-il notamment enlever quelques briques de ce qu'il reste du vrai mur de briques qui encerclait le Ghetto de Varsovie pour les placer à Washington ? Semblablement, par un accord spécial avec le musée d'Auschwitz, des amoncèlements de valises, de parapluies, de brosses à dents, de chaussures, d'assiettes, de prothèses, de boites de Zyklon B ont été fournis au musée de Washington. Régine Robin raconte les terribles dilemmes que soulèvent à la fois l'importation des mémoires, d'artefacts et de reliques humaines.

Il devait en outre, être question de neuf kilos de cheveux, mais la discussion fut très vive pour savoir si le déploiement des cheveux humains venus d'Auschwitz dans l'exposition permanente était licite ou non. Comment dans ce cadre aseptisé, exposer ces reliques? La discussion s'éternisa. Le comité passa aux votes et par 9 voix contre 4, décida de placer les cheveux d'Auschwitz dans l'exposition permanente. Certains poussèrent cependant au réexamen de la question. Finalement, devant l'argument que ce déploiement pourrait heurter l'identité féminine de certaines survivantes, qu'on pourrait se demander si ces cheveux n'étaient pas ceux d'un membre de leur famille, il fut décidé

\footnotetext{
${ }^{14}$ A l'époque où il a été retenu comme architecte de ce Mémorial, il était l'un des principaux associés de l'architecte leoh Ming Peï, concepteur entre autre de la pyramide du Louvre (1988).

${ }^{15} 90 \%$ des visiteurs ne sont pas Juifs, d'après le site du musée : http://www.ushmm.org/

${ }^{16}$ Lanzmann a répondu à cette question en ne montrant pas une seule image d'archives dans son film Shoah.
} 
qu'on laisserait ces kilos de cheveux à Auschwitz, quelque part dans les entrepôts de l'oubli et qu'on se contenterait de prendre des photos des vitrines du vrai musée d'Auschwitz. (Robin, 1998).

\section{Chacun... Et tous les autres, d'un point de vue idéel et matériel}

En effet, comment "faire avec " cette histoire et ces mémoires traumatiques, avec ces et ses émotions ? Ces dernières sont ressenties de manière individuelle mais s'insèrent dans un entrelacs de normes et de partages qui appartiennent au/à des collectif(s). Les émotions singulières et personnelles co-agissent, co-habitent et se confrontent aux affects des autres visiteurs.

Qu'est-ce qu'un musée de mémoires douloureuses, si ce n'est finalement un lieu où les émotions circulent, se frottent les unes aux autres et se confrontent aux questions des rapports de pouvoirs, de normes, de langages verbaux et corporels ? Les mémoires des victimes de dictatures, de totalitarisme ou de génocides réactivent aussi des dimensions personnelles, à la fois travaillées par des souvenirs amicaux ou familiaux et hantées par l'absence, la perte et la souffrance. Mais ces émotions, forcément singulières, sont aussi proposées au partage. Les multiples objets, les photographies individuelles, les listes de noms rappellent ce désir de s'extraire d'une mort de masse, et de se réapproprier la mort. II s'agit de la reconnaissance d'un " je " qui s'inscrit dans un "nous ». Les expositions de photographies, et notamment de photographies de visages, occupent toujours une place centrale dans le dispositif muséal, qu'il s'agisse du musée national du 11-Septembre qui a ouvert ses portes à Ground Zero, à New York, en mai 2014 (Truc, 2015), des musées et mémoriaux consacrés à la Shoah, du musée S21 à Phnom Penh...

Les rituels d'évocation des noms jouent également un rôle central et partagé en tant que canalisateurs d'émotions. Aux morts sans cérémonie et sans sépulture, l'inscription ou l'énonciation du nom contribuent à redonner identité et patronyme, en leur nom propre. À Yad Vashem, la Salle des Noms joue ce rôle de pierre tombale symbolique. Les noms sont inscrits dans le Dôme des Noms mais aussi sur le site internet où chacun peut consulter et retrouver les traces d'un aïeul, d'un oncle, d'un parent ${ }^{17}$. Le web-documentaire réalisé par Camille Clavel, "Vers où Israël » ${ }^{18}$, commence justement par une quête du réalisateur auprès du musée de Yad Vashem.

Noms des victimes, nom des Justes, ou noms des personnes rescapées comme au musée des réfugiés juifs de Shanghai, leur inscription matérielle octroie une matérialité au statut de (victime, Juste ou rescapé) et restitue à chacun son individualité. Nombreux sont les visiteurs qui touchent, effleurent ou caressent ces noms, du bout des doigts (Figure 9).

\footnotetext{
${ }^{17}$ www.yadvashem.org

${ }^{18}$ http://next.liberation.fr/cinema/2012/11/01/vers-ou-israel 868428
} 
Figure 9 : Touriste devant l'inscription des Noms du mémorial Reflecting Absence. Site du Mémorial du 9/11. New York

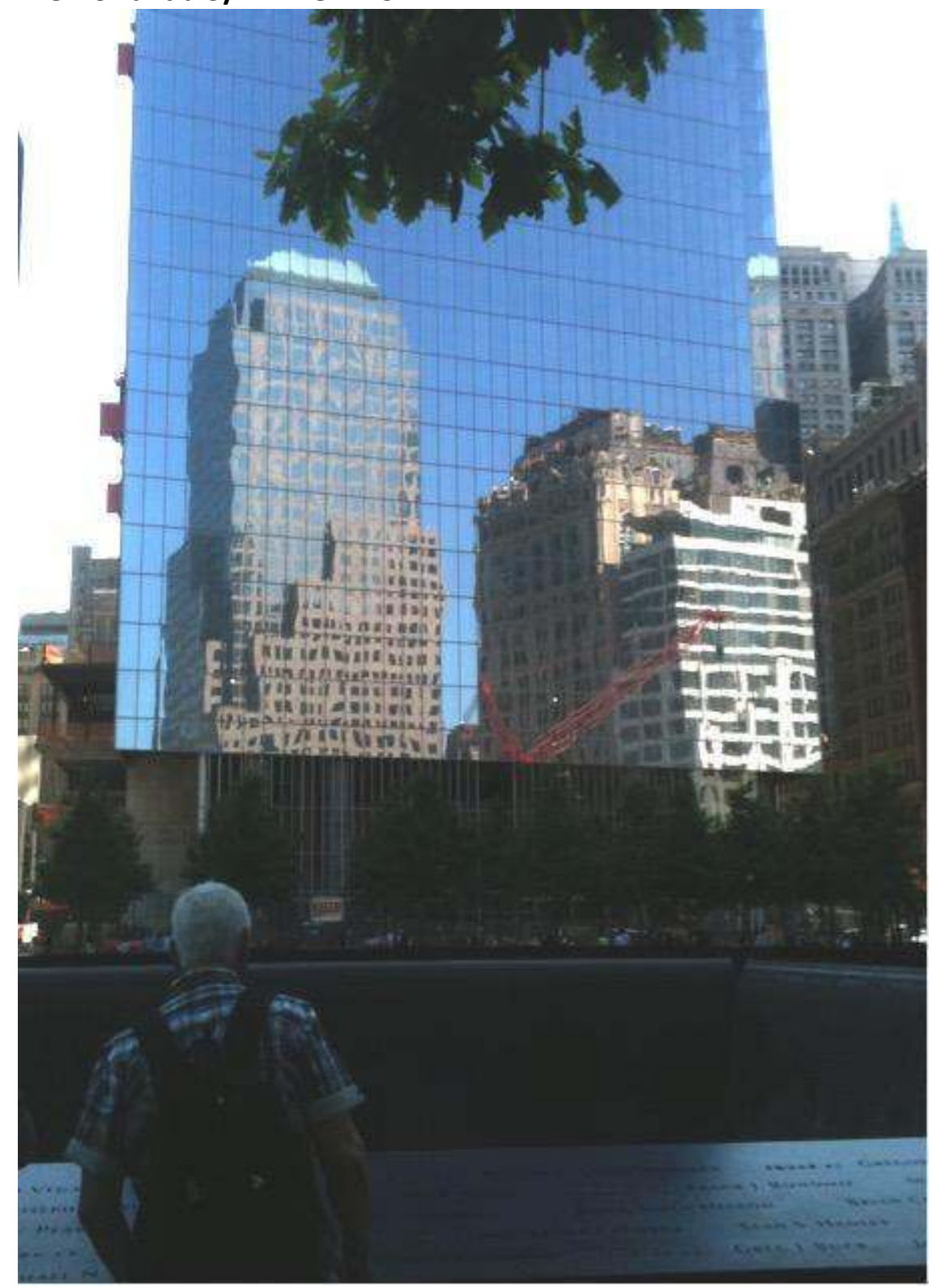

Cliché : Dominique Chevalier, mai 2012.

À Ground Zero, qui commémore le traumatisme du 11 septembre 2001 selon des dispositions très proches des modalités propres à la mémoire de la Shoah, l'inscription des noms des victimes des attentats a suscité quelques polémiques et montre ce qu'au-delà de la commémoration des noms, l'inscription exprime en termes de proximité interhumaine. Les Reflecting Pools, deux fontaines de 4000 mètres carrés chacune, bornées par un parapet en bronze sur lequel les noms des victimes sont gravés, ont été installées à l'emplacement des Twin Towers (Truc, 2015). Les architectes du projet, Micheal Arad et Peter Walker, avaient initialement prévu de disposer les noms de manière aléatoire, mais sous la pression des familles et proches des défunts, ils ont révisé leur choix et décidé d'assembler les noms en fonction des relations sociales que les victimes entretenaient avant les attentats. Deux ans de recherche et d'enquêtes ont été nécessaires pour arriver à un "bon voisinage » matériel et mémoriel entre maris et femmes, frères et sœurs, collègues et amis. 
À Berlin, le Centre d'Informations attenant au Mémorial d'Eisenman (ajouté au projet initial), renferme également une Salle des Noms. Les noms et les courtes biographies des victimes sont récités par des voix qui emplissent la pièce. Un commentaire souligne que " La lecture des noms et des courtes biographies de l'ensemble des six millions de victimes, sous cette forme, prendrait environ six ans, sept mois et vingt-sept jours ". Ici, comme au Mémorial des Enfants de Yad Vashem où une voix, en fond sonore, rappelle, dans une litanie sans fin, les prénoms, noms, âges et pays d'origine des enfants assassinés, en anglais, en hébreu et en yiddish, la tristesse, l'angoisse, la consternation sont palpables. Des sanglots, des reniflements entrecoupent régulièrement le récitatif de la voix enregistrée.

Cette lecture des noms s'accomplit chaque année pour Yom Ha Shoah. Au Mémorial de la Shoah à Paris, par exemple, une lecture publique ininterrompue de 24 heures se tient devant le Mur des Noms sur lequel sont gravés les 76000 noms de juifs de France déportés par convois. Environ deux cents personnes, anonymes, bénévoles, anciens déportés, personnalités, se relaient pour lire les noms de "ceux dont il ne reste que le nom " pour reprendre l'expression de Simone Veil, lors de son discours inaugural du Mur des Noms du Mémorial de la Shoah de Paris, le 23 janvier 2005. À New York, cette lecture des noms a été l'un des moments forts de la cérémonie d'inauguration des Reflecting Pools, en 2011.

Au récitatif de la liste de chaque nom répond une émotion individuelle dont la co-présence doit elle aussi induire, symétriquement, un collectif. C'est dans ce croisement des individus, les disparus et les vivants, qu'une mémoire collective doit s'élaborer. La mémoration de chaque victime est sensée produire une commémoration partagée. Enfin, tel est le projet de ces lieux, dans leur muséographie.

Mais ces musées, au delà de leurs objets, participent plus largement de stratégies métropolitaines et territoriales, pour lesquelles les dimensions culturelles sont déterminantes. La reconnaissance labellisée accompagne ainsi les lieux de mémoire, quelle qu'en soit l'échelle. Si Auschwitz est classé au Patrimoine mondial de l'UNESCO, d'autres le sont au regard de classements nationaux. L'offre touristique s'est indéniablement enrichie de ces lieux de visites, selon des trajectoires qui conjuguent inflation patrimoniale et mémorielle, construction nationale identitaire et mise en tourisme dont les offres doivent continûment se compléter pour assurer les revenus attendus de cette activité.

Les musées urbains consacrés à la Shoah, édifiés hors de toute proximité avec les camps et centres de mise à mort, participent de relations complexes où " patrimoine " et " tourisme " se reconfigurent en permanence sous le double effet d'un régime d'historicité présentiste (Hartog, 2003) et d'une métropolisation concurrentielle. On peut même considérer que la co-mémoration de la Shoah constitue un réseau mondial, muséal et de sites.

Dès lors, les résonnances de lieux à lieux traumatiques s'effectuent également par l'intermédiaire des événements et expositions. Le musée de l'Holocauste à Washington (USHMM) propose ainsi des expositions sur le génocide cambodgien et sur la perpétration actuelle de génocides comme le propose le centre de la résistance et de la déportation a Lyon (CHRD) qui dédie maintenant depuis plusieurs années des expositions temporaires consacrées aux génocides perpétrés depuis la Seconde Guerre mondiale.

L'ensemble de ces offres, aujourd'hui mises sur le marché des destinations touristiques, soulève évidemment la question du ressort de l'attractivité. Les Rencontres de la photographie d'Arles 2015 ont ainsi exposé I was here, tourisme de la désolation du 
photographe Ambroise Tézenas (Figure 10), qui a effectué les circuits d'un nouveau genre proposé par des tour-opérateurs ${ }^{19}$.

\section{Figure 10 : Exposition I was here, tourisme de la désolation, Rencontres d'Arles}

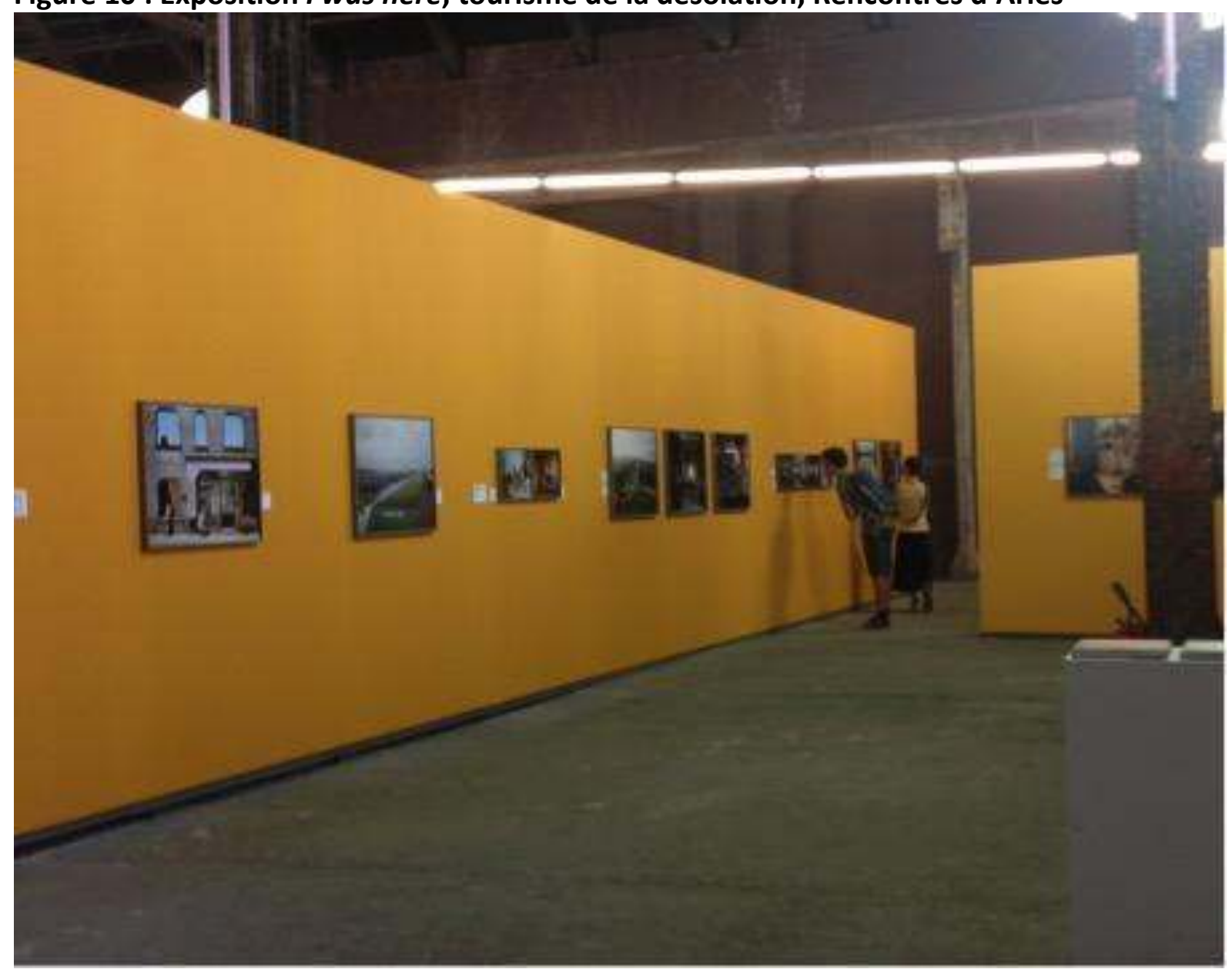

Cliché : Dominique Chevalier, août 2015.

On pourrait rapprocher ce témoignage contemporain en images de pratiques plus anciennes; le goût des émotions fortes n'est ni nouveau, ni dénué d'ambiguïté sur le plan culturel et l'appétit mortifère sert depuis longtemps de motivation touristique (Lennon, Foley, 2014) qu'illustrait déjà, à la fin du XIX ${ }^{e}$ siècle, le succès parisien des visites à la Morgue (Bertherat, 2014).

Même s'il faut, sans doute, établir une distinction entre tourisme de mémoire douloureuse et dark tourism dans leurs rapports à la fabrique mémorielle, une constante de pratiques les recouvre: le projet et l'effectivité des émotions. Se déplacer, parcourir des milliers de kilomètres pour éprouver des émotions liées à la mort. De ce point de vue, l'activité touristique constitue un opérateur mondialisé de sensations fortes et de vertige, forme d'illynx, pour reprendre une des quatre catégorisations de Roger Caillois (1958) et de mise en danger par procuration temporelle. Si le lieu est effectivement celui du danger, le moment ne l'est plus, et d'une certaine manière, la présence (touristique) même atteste qu'il ne l'est plus.

\footnotetext{
${ }^{19}$ En savoir plus sur http://www.lemonde.fr/arts/video/2015/07/10/le-dark-tourism-une-nouvelle-tendancede-voyage 4678682 1655012.html
} 
Pour les lieux de mémoires douloureuses, et parce qu'il s'agit justement de temps passés (depuis plus ou moins longtemps), la présence, touristique, semblerait attester d'un dépassement d'événements qui, parce qu'ils sont recyclés dans une pratique " récréative » ne sauraient plus se reproduire. Les voir et les éprouver, consigneraient leur achèvement et leur non reproductibilité. Tablant ainsi sur l'effet du lieu, sur un effet qui affecte, la visite garantirait un (illusoire) " plus jamais "... C'est, en forme de récit - de mémoire donc - la conviction que l'épreuve émotionnelle en assurerait l'inculcation.

\section{Conclusion}

Le rapport émotionnel aux lieux d'histoire et de mémoires douloureuses constitue un opérateur qui transmute l'une et l'autre pour les confondre dans une alchimie individuelle et collective, celle d'une relation, présente et présentielle, au passé. Relation, c'est-à-dire à la fois, lien, récit et témoignage. Dans ces lieux, la fabrique de la mémoire s'effectue au grain le plus fin, celui de chacun, mais toujours avec et à côté des autres. Le tourisme y continue dès lors d'être ce qu'il est culturellement et politiquement depuis ses prémices, une mobilité productrice de mémoire(s) et d'identité(s). Parce qu'ils sont devenus des lieux touristiques, ces sites historiques et ces musées constituent une "circonstance ", c'est-à-dire au sens propre, un "milieu» pour la mémoire, pour une passation intergénérationnelle, qui s'effectue de moins en moins par l'expérience transmise - Erfharung - mais par l'expérience vécue - Erlebnis - (Benjamin, 1989). L'émotion - les émotions - sont dès lors le vecteur par lequel la passation relève le défi de la mémoire de l'Histoire: "Si ce qu'ils [les lieux de mémoire] défendent n'était pas menacé, on n'aurait plus besoin de les construire. Si les souvenirs qu'ils enferment, on les vivaient vraiment, ils seraient inutiles » (Nora, 1984).

\section{Bibliographie}

APPADURAI A. (2007), Géographie de la colère. La violence à l'âge de la globalisation, Paris, Payot.

BENJAMIN W. (1989), Paris, capitale du XIXe siècle, Paris, Éditions du Cerf.

BERTHERAT B. (2014), " Le kaléidoscope des émotions. L'exposition publique des cadavres à la morgue à Paris au XIX ${ }^{e}$ siècle ", in Ambroise-Rendu A.-C., Demartini A.-E., Eck H., Edelman N. (dir.), Émotions contemporaines. $\mathrm{XIX}^{\mathrm{e}}-\mathrm{XXI}^{\mathrm{e}}$ siècles, Paris, Armand Colin, Collection Recherches.

CABASSET-SEMEDO C., PEYVEL E., SACAREAU I., TAUNAY B. (2010), " De la visibilité à la lisibilité: le tourisme domestique en Asie ", Espace populations sociétés, n²010/2-3, pp. 221-235.

CAILLOIS R. (1958), Les Jeux et les Hommes, Paris, Gallimard.

CAUNE J. (1999), Pour une éthique de la médiation, le sens des pratiques culturelles, Grenoble, Presses universitaires de Grenoble, Collection Communication, médias et sociétés, 294 p.

CHAUMONT J.-M. (1997), La Concurrence des victimes. Génocide, identité, reconnaissance, Paris, La Découverte, rééd. 2002.

CHEVALIER D. (2012), Musées et musées-mémoriaux urbains consacrés à la Shoah: mémoires douloureuses et ancrages géographiques. Les cas de Berlin, Budapest, Jérusalem, 
Los Angeles, Montréal, New York, Paris, Washington, Habilitation à Diriger des Recherches soutenue à l'université Paris 1 Panthéon-Sorbonne.

CHEVALIER D. (2015), "Sunt lacrymae rerum et mentem mortalia tangunt. Quels objets pour signifier la Shoah ? », Géographie et Culture, n91-92, pp. 65-82.

CHEVALIER D. (2015), "Les mémoires douloureuses appréhendées comme ressources patrimoniales. Quels impacts sur les territoires ? " in Spindler J. (dir.), Le tourisme de mémoire : un atout pour les collectivités territoriales ?, Paris, L'Harmattan, pp. 101-116.

GIBLIN B. (2007), " Le tourisme ; un théâtre géopolitique ? ", Hérodote, n 127, pp. 3-14.

HALBWACHS M. (1947), L'expression des émotions et la société, mis en ligne: http://dx.doi.org/doi:10.1522/cla.ham.exp1

HARTMANN R. (2014), "Dark tourism, thanatourism, and dissonance in heritage tourism management ", Journal of Heritage Tourism, vol. 9, n²/2014, pp. 166-182.

HARTOG F. (2003), Régimes d'historicité, Présentisme et expériences du temps, Paris, Le Seuil.

HERTZOG A. (2012), "Tourisme de mémoire et imaginaire touristique des champs de bataille », Via@, $n^{\circ} 1 / 2012$, mis en ligne le 16 mars 2012. URL : http://www.viatourismreview.net/Article6.php

LENNON J. J. (2014), "Tourisme noir. Le goût pour la mort et le désastre ", in Tézenas A., Tourisme de la désolation, Arles, Actes Sud.

LENNON J. J., FOLEY M. (2000), Dark Tourism. The Attraction of Death and Disaster, Londres, Continuum.

LEVI P. (1987), Si c'est un homme, Paris, Julliard, collection Pocket.

LIBESKIND D. (2005), Construire le futur d'une enfance polonaise à la Freedom Tower, Paris Albin Michel.

NORA P., (1984), "Entre mémoire et histoire, la problématique des lieux ", in Nora P. (dir.), Les lieux de mémoire, t.1, Paris, Gallimard, p. XXIV.

PEREC, G., (1975), W ou le souvenir d'enfance, Paris, Denoël.

RICOEUR P., (2000), La mémoire, I'histoire, l'oubli, Paris, Le Seuil.

ROBIN R. (1998), «La mémoire saturée », en ligne: http://raicc.mcgill.ca/raicc\%20accueil fichiers/moscov98.htm, page consultée le 16 février 2010.

ROSSET C., (1977), Le Réel : Traité de l'idiotie, Paris, Éditions de Minuit.

TRAVERSO E., (2009), "L'Europe et ses mémoires. Trois perspectives croisées. ", Raisons politiques, $n^{\circ} 36, \mathrm{pp}$. 151-167.

TRUC G. (2015), "Venir à Ground Zero. Se souvenir du 11-septembre », Espacetemps.net, rubrique Travaux, 28 avril 2015, http://www.espacestemps.net/articles/venir-a-groundzero-se-souvenir-du-11-septembre/

URBAIN J.-D., (2003), « Tourisme de mémoire. Un travail de deuil positif », Cahiers Espaces, nº ${ }^{\circ 0}$, pp. 5-7. WEIZMANN, D., (2005), « Auschwitz : lieu de mémoire ou lieu de négation? », in Boursier J.-Y. (dir.), Musées de guerre et mémoriaux, Paris, Editions de la Maison des Sciences de l'Homme.

WIEVIORKA A. (1998), L'Ère du témoin, Paris, Plon, 189 p.

WIEVIORKA A. (2011), L'heure d'exactitude. Histoire, mémoire, témoignage, Paris, Albin Michel, $256 \mathrm{p}$.

YOUNG J. E. (1991), "Holocaust Memorials in America: The Politics of Identity ", Survey of Jewish Affairs, pp.161-173. 